\title{
White-light diffraction tomography of unlabelled live cells
}

\author{
Taewoo Kim ${ }^{1 \dagger}$, Renjie Zhou ${ }^{1,2 \dagger}$, Mustafa Mir', S. Derin Babacan³, P. Scott Carney4, Lynford L. Goddard ${ }^{2}$ \\ and Gabriel Popescu ${ }^{1 \star}$
}

\begin{abstract}
We present a technique called white-light diffraction tomography (WDT) for imaging microscopic transparent objects such as live unlabelled cells. The approach extends diffraction tomography to white-light illumination and imaging rather than scattering plane measurements. Our experiments were performed using a conventional phase contrast microscope upgraded with a module to measure quantitative phase images. The axial dimension of the object was reconstructed by scanning the focus through the object and acquiring a stack of phase-resolved images. We reconstructed the threedimensional structures of live, unlabelled, red blood cells and compared the results with confocal and scanning electron microscopy images. The $350 \mathrm{~nm}$ transverse and $900 \mathrm{~nm}$ axial resolution achieved reveals subcellular structures at high resolution in Escherichia coli cells. The results establish WDT as a means for measuring three-dimensional subcellular structures in a non-invasive and label-free manner.
\end{abstract}

A transparent object illuminated by an electromagnetic field generates a scattering pattern that carries specific information about its internal structure. Inferring this information from measurements of the scattered field, that is, solving the inverse scattering problem, is the fundamental principle that has allowed X-ray diffraction measurements to reveal the molecular-scale organization of crystals ${ }^{1}$ and more recently, image cells with nanoscale resolution ${ }^{2,3}$. The scattered field is related to the spatially varying dielectric susceptibility of the scattering object by a transformation that simplifies considerably and, more importantly, becomes invertible, when the incident field is only weakly perturbed by the presence of the object. In this regime, the first-order Born approximation ${ }^{4}$ and the Rytov approximation ${ }^{5}$ have been used to unambiguously retrieve the three-dimensional spatial distribution of the dielectric constant. Implementation of inverse scattering requires knowledge of both the amplitude and phase of the scattered field. This obstacle, known as the phase problem, has been associated with X-ray diffraction measurement throughout its century-old history (for a review, see ref. 6).

In 1969, Wolf proposed diffraction tomography as a reconstruction method combining the X-ray diffraction principle with optical holography ${ }^{7}$. Unlike X-rays, light at lower frequencies can be used in phase imaging measurements, as demonstrated by Gabor ${ }^{8}$. In recent years, as a result of new advances in light sources, detector arrays and computing power, quantitative phase imaging (QPI), in which optical path-length delays are measured at each point in the field of view, has become a very active field of study? Whether involving holographic or non-holographic methods ${ }^{10-16}$, QPI presents new opportunities for studying cells and tissues non-invasively, quantitatively and without the need for staining or tagging ${ }^{17-23}$. Projection tomography using laser QPI has made use of ideas from X-ray imaging and enabled three-dimensional imaging of transparent structures ${ }^{24-26}$. More recently, this method has been applied to live cells ${ }^{27-30}$. This type of reconstruction has a complex set-up because of the requirement to either scan the illumination angle or rotate the specimen about a fixed axis. As a result, this method is limited to shallow depths of field ${ }^{31}$. Importantly, without additional efforts such as synthetic aperture ${ }^{30}$ and digital de-noising techniques ${ }^{32}$, laser light imaging is plagued by speckles, which ultimately limit the resolving power of the method ${ }^{33}$. To mitigate this problem, tomographic methods based on white light have also been proposed ${ }^{34-36}$. These approaches require a priori knowledge of the three-dimensional point spread function (PSF) of the instrument and ignore the physics of the light-specimen interaction. Despite these efforts, three-dimensional cell imaging is still largely restricted to confocal fluorescence microscopy, an invasive method ${ }^{37}$.

Here, we report on a new approach for label-free tomography of live cells and other transparent specimens, which we refer to as white-light diffraction tomography (WDT). WDT offers a high-performance, simple design, as well as suitability for operation in a conventional microscopy setting. Its main features can be summarized as follows. First, WDT is a generalization of diffraction tomography to broadband illumination. Second, WDT operates in an imaging rather than a scattering geometry. Note that this is a departure from the far-zone, angular scattering that is traditionally used in $\mathrm{X}$-ray diffraction. When dealing with transparent objects, measuring the complex field at the image plane yields higher sensitivity than measuring in the far-zone ${ }^{38}$. Third, WDT is implemented using an existing phase contrast microscope with white-light illumination, and the three-dimensional structure is recovered by simply translating the objective lens, which scans the focal plane axially through the specimen. Because phase contrast microscopes are commonly used, the method shown here could be adopted on a large scale by non-specialists.

\footnotetext{
'Quantitative Light Imaging Laboratory, Department of Electrical and Computer Engineering, Beckman Institute for Advanced Science and Technology, University of Illinois at Urbana-Champaign, Urbana, Illinois 61801, USA, ${ }^{2}$ Photonic Systems Laboratory, Department of Electrical and Computer Engineering, Micro and Nanotechnology Laboratory, University of Illinois at Urbana-Champaign, Urbana, Illinois 61801, USA, ${ }^{3}$ Beckman Institute for Advanced Science and Technology, University of Illinois at Urbana-Champaign, Urbana, Illinois 61801, USA, ${ }^{4}$ Department of Electrical and Computer Engineering, Beckman Institute for Advanced Science and Technology, University of Illinois at Urbana-Champaign, Urbana, Illinois 61801, USA, 'These authors contributed equally to this work. ${ }^{\star}$ e-mail: gpopescu@illinois.edu
} 

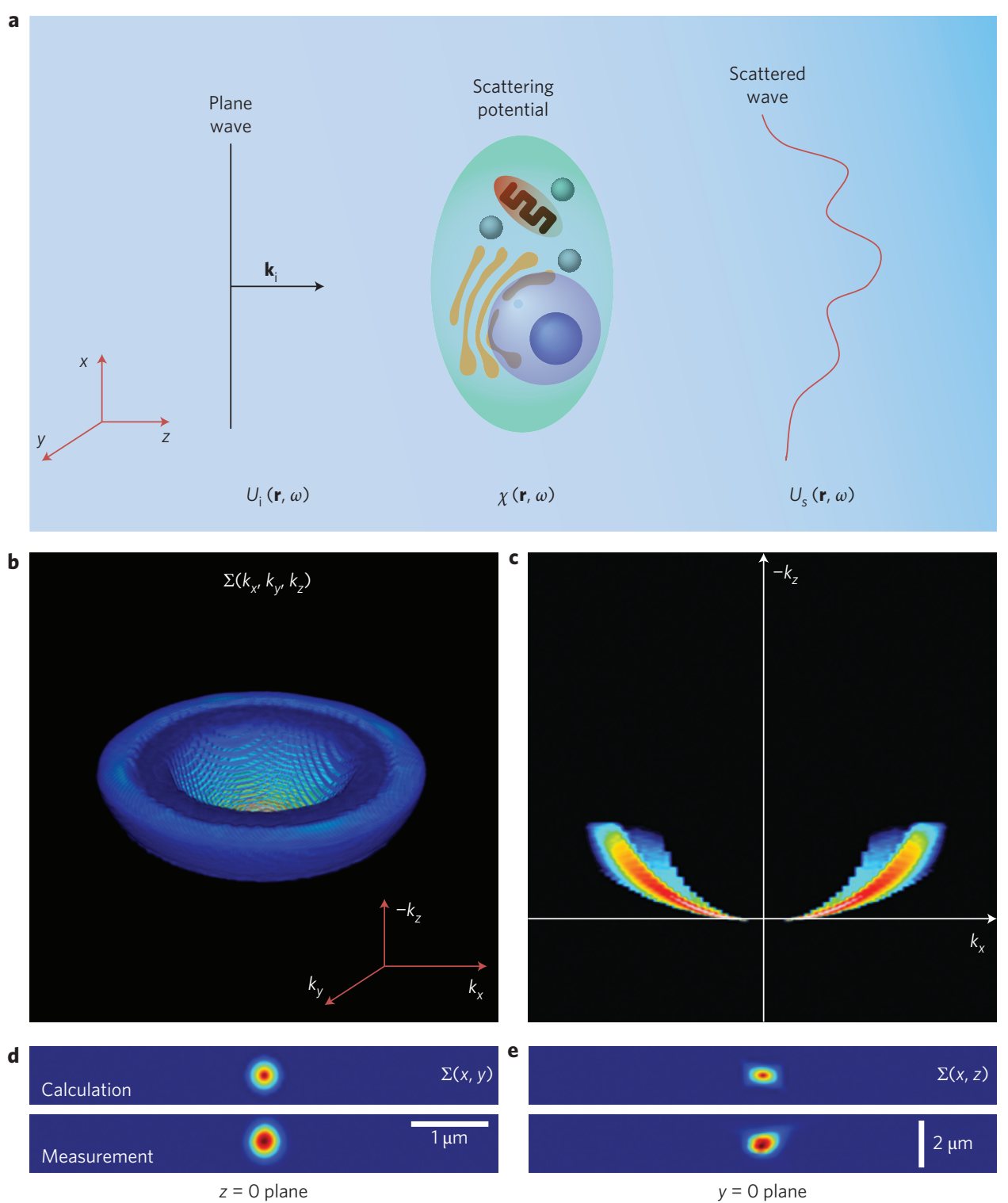

Figure 1 | The scattering problem. a, Illustration of light scattering under the first-order Born approximation where a plane wave's wavefront is perturbed by the object. $\mathbf{b}$, Three-dimensional rendering of the instrument transfer function, using the proposed WDT calculation. c, Cross-section of the transfer function at the $k_{y}=0$ plane. $\mathbf{d}$, Calculated and measured PSF at the $z=0$ plane. e, Calculated and measured PSF in the $y=0$ plane.

\section{Tomographic reconstruction and resolution}

It is known that the Rytov approximation is more appropriate for reconstructing smooth objects with respect to the wavelength of light, that is, for low-resolution imaging, and the Born approximation works better for imaging finer structures (see, for example, ref. 39, p. 485). Accordingly, we use the latter here. Under the first-order Born approximation (Fig. 1a) with an incident plane wave $U_{\mathrm{i}}=A(\omega) e^{i \beta(\omega) z}$, we solve the forward scattered field $U_{s}$ in the wavevector space instead of using the traditional Green's function and Weyl's formula approach (see Methods and Supplementary Section b). In the transverse wavevector domain $\mathbf{k}_{\perp}, U_{\mathrm{s}}$ can be expressed as

$$
U_{s}\left(\mathbf{k}_{\perp}, z ; \omega\right)=-\frac{\beta_{0}^{2}(\omega) A(\omega) e^{i q z}}{2 q} \chi\left[\mathbf{k}_{\perp}, q-\beta(\omega)\right]
$$

where $\beta(\omega)=\bar{n} \beta_{0}(\omega)$, with $\bar{n}$ being the spatial average of the refractive index associated with the object, $\beta_{0}(\omega)=\omega / c$ is the propagation constant (or the wavenumber) in vacuum, $\omega$ is the angular frequency, $\chi$ is the scattering potential of the non-dispersive object, $\quad \chi(\mathbf{r})=n^{2}(\mathbf{r})-\bar{n}^{2} \quad$ and $q=\sqrt{\beta^{2}(\omega)-k_{\perp}^{2}}$. (See Supplementary Section $b$ and, for an alternative derivation ${ }^{7}$.) The dispersion in the object is neglected, because most biological samples of interest here are weakly absorbing. This is true even for single red blood cells (RBCs). Even though haemoglobin absorbs strongly in blue, the overall absorption of visible light through a single RBC is very small. This is so because the absorption length of haemoglobin in a normal $\mathrm{RBC}$ is $\sim 10 \mu \mathrm{m}$ in the blue (averaged over wavelengths of $400-500 \mathrm{~nm}$ ) and $\sim 3 \mathrm{~mm}$ in the red (averaged over wavelengths of $600-750 \mathrm{~nm})^{40}$, while the thickness of the cell is only 2-3 $\mu \mathrm{m}$. More discussion on the dispersion effect through a RBC is presented in the Supplementary Section i.

Note that, throughout this Article, we use the same symbol for a function and its Fourier transform. To indicate the domain in which the function operates, we carry all the arguments explicitly; for example, $f\left(\mathbf{k}_{\perp}, z ; \omega\right)$ is the Fourier transform of $f\left(\mathbf{r}_{\perp}, z ; t\right)$ over $\mathbf{r}_{\perp}$ and $t$. In conventional phase shifting interferometry, the crosscorrelation of the scattered and reference fields is measured as 
a
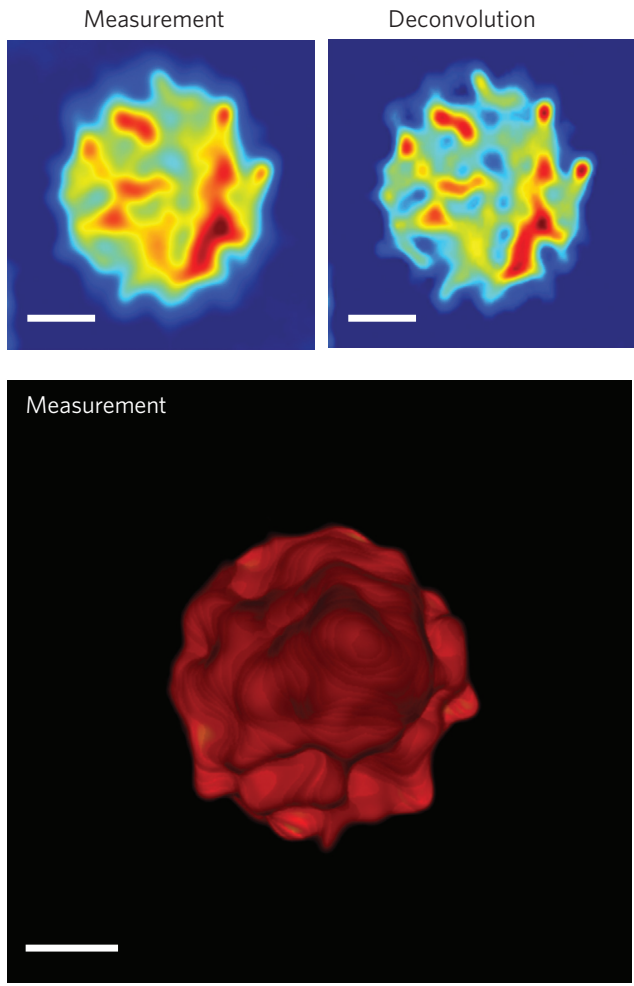

c Measurement
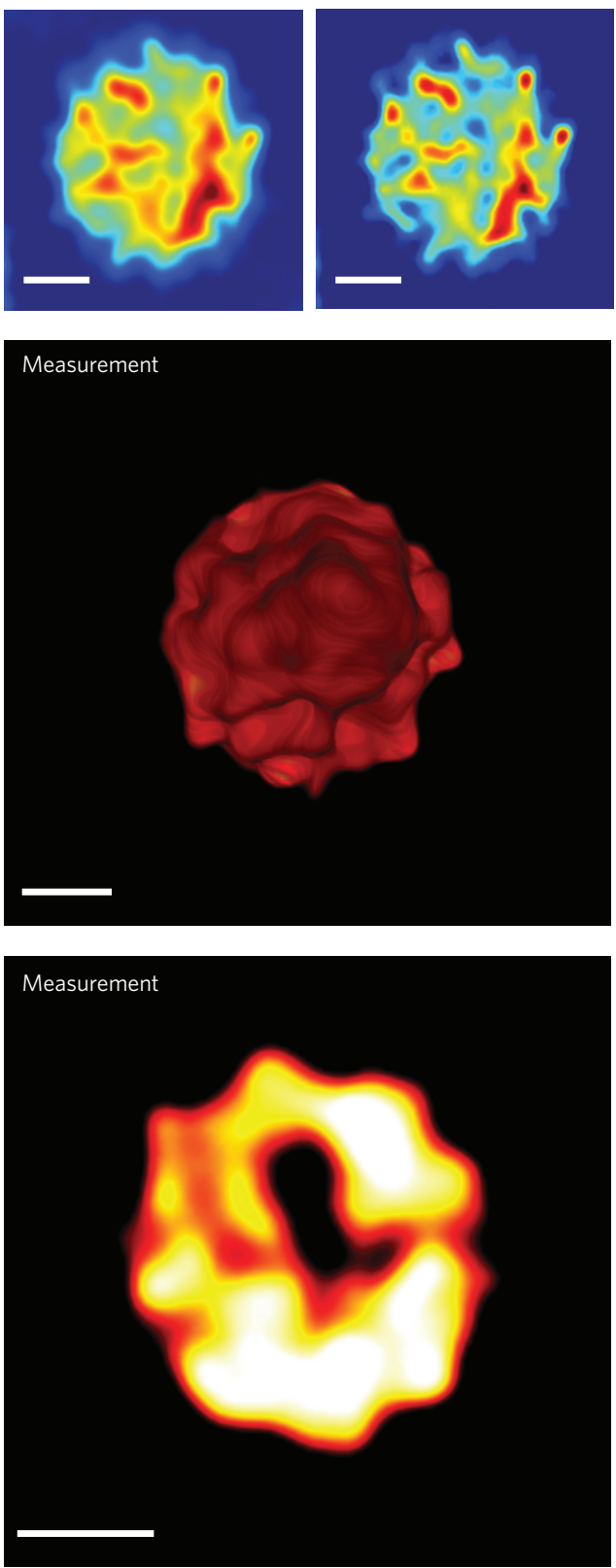

SEM

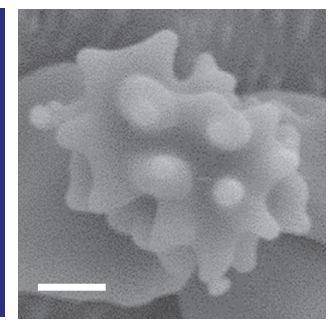

Deconvolution

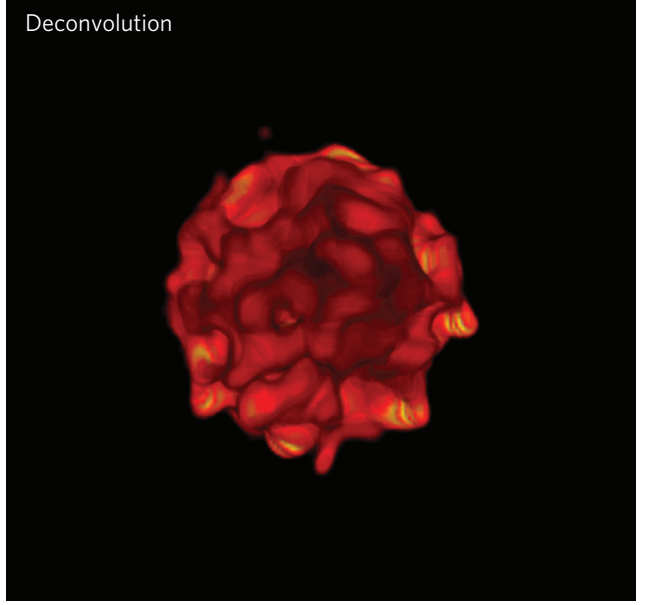

Deconvolution

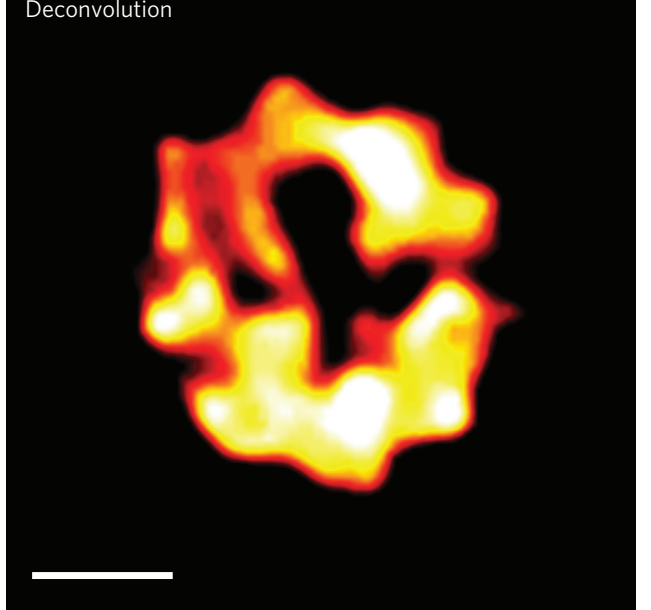

Figure 2 | WDT of RBCs. a, Measured z-slice of a spiculated RBC and the corresponding deconvolution using a $\times 40 / 0.75$ NA objective. An SEM image and a confocal of similar cells are shown for comparison. $\mathbf{b}$. Three-dimensional rendering of the raw data and the corresponding three-dimensional deconvolution (Supplementary Movie 1). c, A measured z-slice and the corresponding deconvolution result, showing the empty space between spicules on the RBC. Scale bars, $2 \mu \mathrm{m}$ in space. The reconstruction uses a z-stack of 100 images, each with $128 \times 128$ pixels, which requires about 5 min for sparse deconvolution.

$\Gamma_{12}\left(\mathbf{r}_{\perp}, z, \tau\right)=\left\langle U_{s}\left(\mathbf{r}_{\perp}, z, t\right) U_{\mathrm{r}}^{*}(z, t+\tau)\right\rangle$ at $\tau=0$, which is equivalent to integrating the cross spectral density over $\omega$. Knowledge of the spatial frequency response of our instrument, or coherent transfer function (see Supplementary Section c) $\Sigma\left(k_{x}, k_{y}, k_{z}\right)$, allows us to write the main result of our calculation (that is, the solution to the inverse scattering problem) in terms of the measured data $\Gamma_{12}$ and the instrument function (or the coherent transfer function) $\Sigma$ in the wavevector domain as

$$
\chi(\mathbf{k})=\frac{\Gamma_{12}(\mathbf{k} ; 0)}{\Sigma(\mathbf{k})}
$$

In practice, the operation in equation (2) requires regularization, as detailed in the Supplementary Section d. Transfer function $\Sigma(\mathbf{k})$ is given by

$$
\Sigma(\mathbf{k})=\frac{1}{8 \bar{n}^{2}} \frac{\left(Q^{2}+k_{\perp}^{2}\right)^{2}}{Q^{3}} S\left(-\frac{Q^{2}+k_{\perp}^{2}}{2 Q}\right)
$$

where $S$ is the optical spectrum of the imaging field as a function of the wavenumber and $Q=\sqrt{\beta^{2}-k_{\perp}^{2}}-\beta$ (see Methods and Supplementary Section b). The three-dimensional PSF can be obtained through an inverse Fourier transform of equation (3).

Qualitatively, $\Sigma$ has a physically intuitive behaviour (Fig. 1b-e). Specifically, its dependence on $z$ is related to the optical spectrum $S(\omega)$ via a Fourier transform, meaning that a broader optical spectrum gives a narrower function, $\Sigma(z)$. This relationship explains the inherent optical sectioning capabilities of the instrument. 

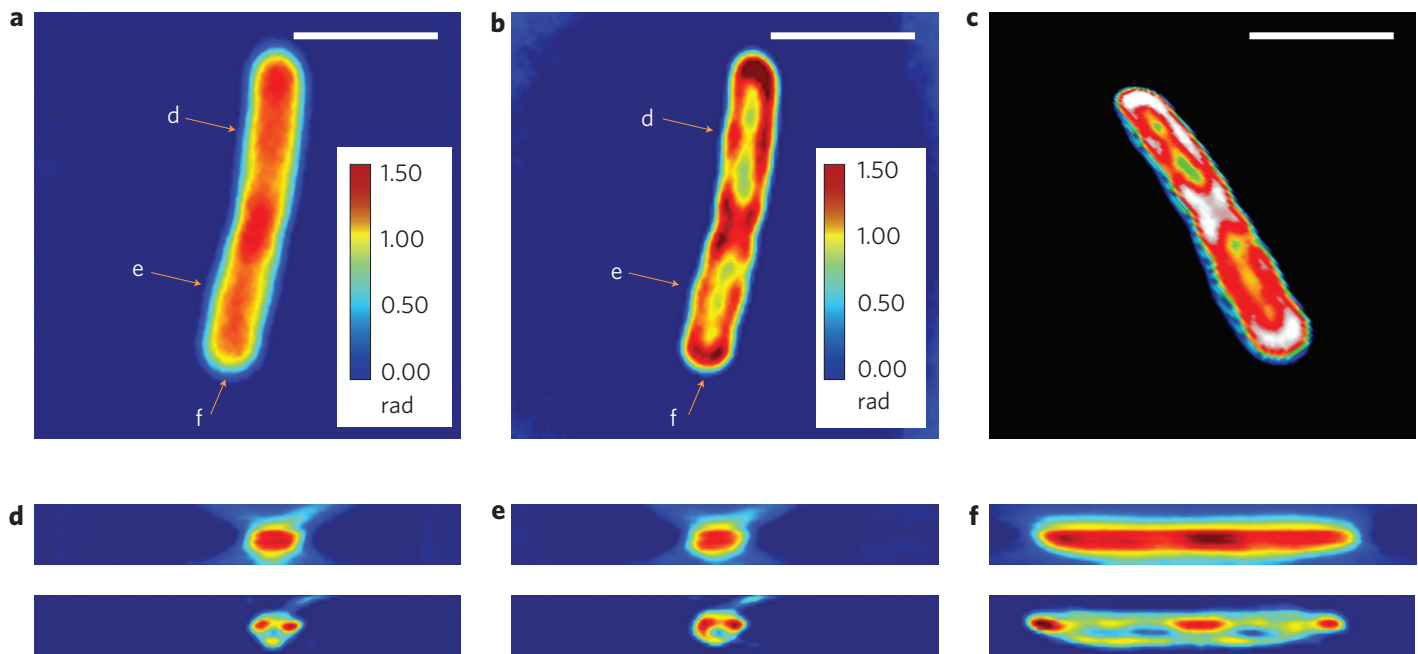

Figure 3 | WDT of $\boldsymbol{E}$. coli cells. a, The centre frame of a $z$-stack measurement using a $\times 63 / 1.4$ NA oil immersion objective. $\mathbf{b}$, Deconvolution result of the same $z$-slice as in $\mathbf{a}$, clearly showing a resolved helical structure. $\mathbf{c}$, Centre cut of the three-dimensional rendering of the deconvolved $z$-stack, which shows both the overall cylindrical morphology and a helical subcellular structure (Supplementary Movie 2). d-f, Cross-sections of the measured $z$-stack (top row) and the deconvolved $z$-stack (bottom row). Each figure label corresponds to the markers shown in $\mathbf{a}, \mathbf{b}$ and is in the same scale as $\mathbf{a}, \mathbf{b}$. Scale bars, $2 \mu \mathrm{m}$. A $z$ stack of 17 images, each with $128 \times 128$ pixels is used for the reconstruction, which requires about 3 min for sparse deconvolution.
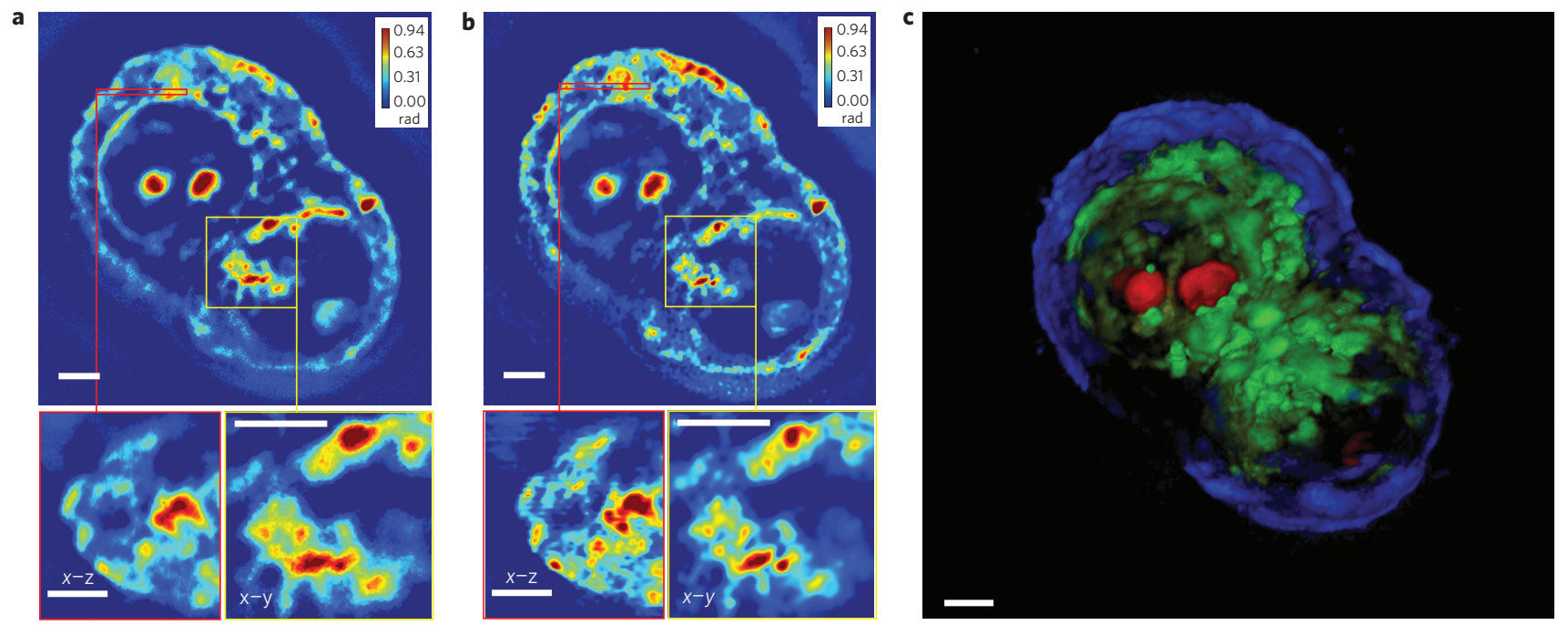

Figure 4 | WDT of HT29 cells. a, A measured z-slice (top), a cross-section at the area indicated by the red box (bottom left) and a zoomed-in image of the area indicated by the yellow box (bottom right), measured using a $\times 63 / 1.4 \mathrm{NA}$ oil immersion objective. $\mathbf{b}$, A deconvolved $z$-slice corresponding to the measurement shown in a (top), a cross-section at the area indicated by the red box (bottom left) and a zoomed-in image of the area indicated by the yellow box (bottom right). By comparing $\mathbf{a}$ and $\mathbf{b}$, the resolution increase can be clearly seen. $\mathbf{c}$, False-colour three-dimensional rendering of the deconvolution result (Supplementary Movie 3). We used z-stacks of 140 images, each with a dimension of $640 \times 640$. Owing to the large image dimension, the image is split into 25 sub-images for faster deconvolution. Overall, the deconvolution process took approximately an hour. Scale bars in all panels, $5 \mu \mathrm{m}$.

This type of optical gating, in which axial resolution is determined by the coherence properties of the illumination light, has been successfully applied in optical coherence tomography (OCT) of deep tissues ${ }^{41,42}$. However, there are significant differences between WDT and OCT. In OCT, the cross-correlation $\Gamma_{12}$ is resolved over a broad delay range, which provides the depth dimension of the object. In WDT, the $z$-information is collected by scanning the focus through the object. Most importantly, in our method, the coherence gating works in synergy with the highnumerical-aperture (NA) optics and thus allows for high-resolution tomography. In other words, in WDT, coherence gating by itself would not work at zero NA and, conversely, high-NA gating would not work with monochromatic light.
We used broadband light from a halogen lamp and high-numerical-aperture objectives $(\times 40 / 0.75 \mathrm{NA}$ and $\times 63 / 1.4 \mathrm{NA})$, resulting in optical sectioning capabilities suitable for high-resolution tomography. Using high-NA objectives, polarization could play a role. However, for weakly scattering, isotropic objects, this effect is negligible. The function $\Sigma\left(k_{x}, k_{y}, k_{z}\right)$ for our imaging system is illustrated in Fig. 1b,c. As expected, the width of the $k_{z}$ coverage increases with $k_{x}$, indicating that the sectioning is stronger for finer structures or, equivalently, higher scattering angles. The structure of the object is recovered through a sparse deconvolution algorithm (see Supplementary Section d). Figure 1d,e shows the transverse and longitudinal cross-sections of the calculated and measured $\Sigma(x, y, z)$, which determine the final resolution. 
a
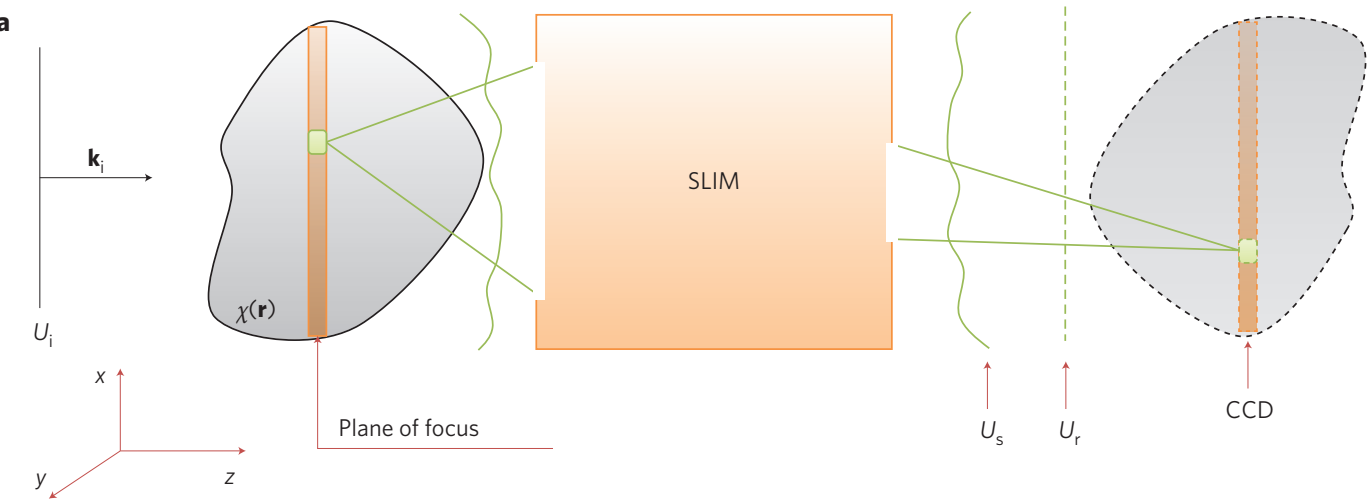

b
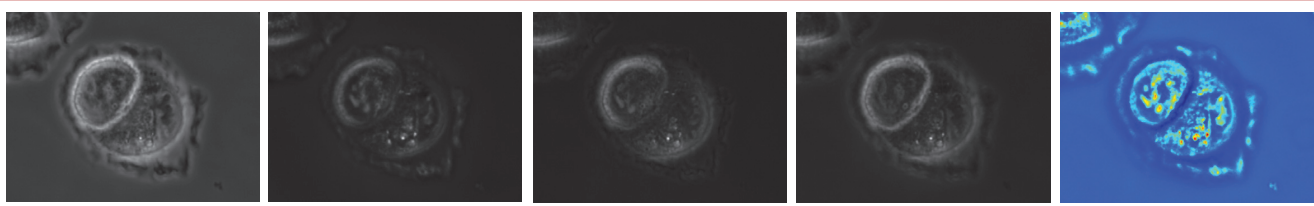

$\pi / 2$

$\pi$

$3 \pi / 2$

0

Phase

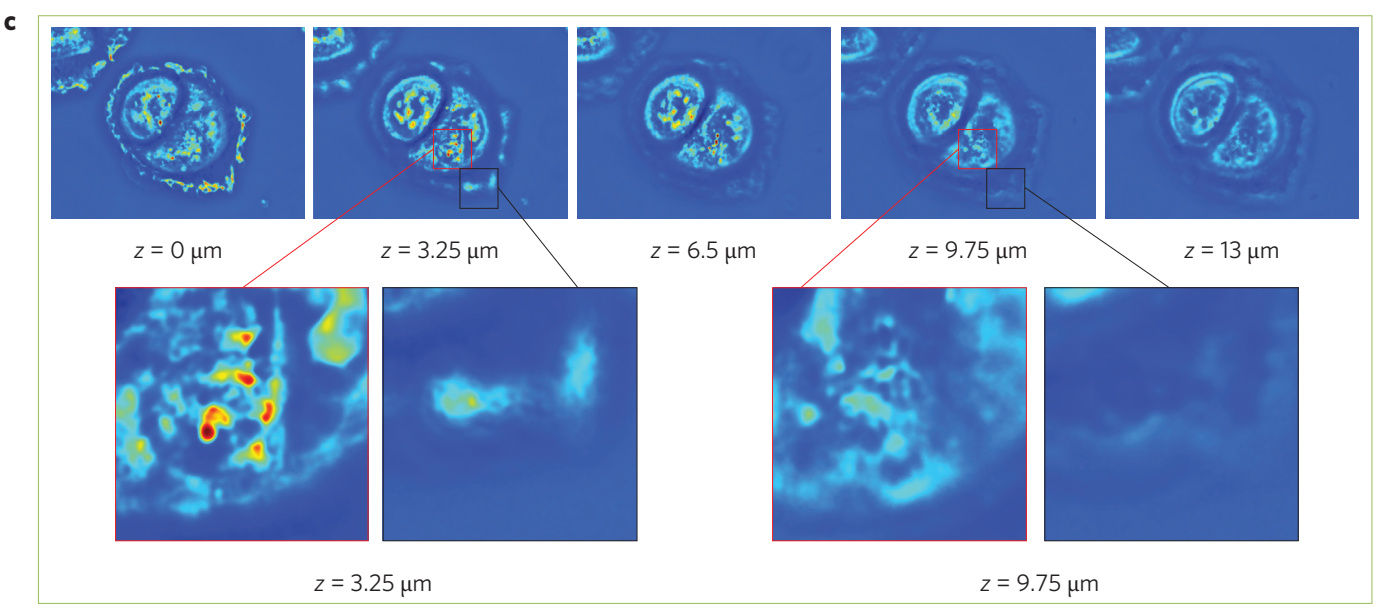

Figure 5 | Illustration of data acquisition. a, Optical sectioning in a phase contrast microscope, where an incident plane wave $U_{i}$ is scattered by an object $\chi$ and the CCD measures the scattered field $U_{s}$ and the reference field $U_{r}$ A detailed description of the SLIM model is provided in Supplementary Fig. 1. b. Example of phase reconstruction using four different phase-shifted intensity images. Applied phase shifts for each image are indicated. c, Example of optical sectioning in SLIM is shown as the focus scans through the U2OS cell over a range of $13 \mu \mathrm{m}$ (top). Red and black outlined regions are zoomed in to more clearly show the optical sectioning ability (bottom). The colour scheme represents the phase value, with red representing large phase values and blue representing small phase values.

Experimentally, $\Sigma$ was measured by imaging a microsphere much smaller than the resolution of the system, as detailed in the Supplementary Section d. The measured and experimental functions show good agreement, with the measured function being slightly larger than the calculations predict $(0.39 \mu \mathrm{m}$ versus $0.35 \mu \mathrm{m}$ transversely; $1.22 \mu \mathrm{m}$ versus $0.89 \mu \mathrm{m}$ longitudinally). This is expected, as the particle used for the measurement has a finite thickness that adds to the width of $\Sigma$.

\section{Tomography of spiculated RBCs}

After validating our WDT method using a polystyrene microbead sample (see Supplementary Section h), we first applied WDT to measure spiculated RBCs, known as echinocytes. This morphological abnormality is well documented and can be an indication of transitory stress (for example, osmotic stress) or a sign of a serious disease $\mathrm{e}^{21,43}$. We used this interesting three-dimensional morphology as a test sample and used a scanning electron microscopy (SEM) image and a confocal fluorescence microscopy image ${ }^{44}$ as control imaging methods (Fig. 2a). The sample was prepared as a blood smear on a glass slide, and phase images were measured using spatial light interference microscopy (SLIM), as described in the Methods. Unlike SEM and confocal microscopy, which require sample preparation steps such as metal deposition and fluorescence labelling (Calcein and DiI in Fig. 2a), WDT is label-free and works without sample preparation. Furthermore, the irradiance at the sample plane in WDT is six to seven orders of magnitude lower than in confocal microscopy ${ }^{16}$, which provides a less harmful environment for the sample. Axial data ( $z$-stack) were acquired in steps of $250 \mathrm{~nm}$ and a precision of $10 \mathrm{~nm}$ was ensured by the piezoelectric nosepiece. With the $\Sigma(x, y, z)$ function computed for a $\times 40 / 0.75$ NA objective, we performed the three-dimensional deconvolution based on the sparsity constraint ${ }^{34,45}$ (see Supplementary Section d). Figure 2a presents a SLIM projection image of an echinocyte and its corresponding deconvolved image, as well as an SEM image of a similar echinocyte. Sharper surface structures are observed for the RBC in the deconvolved image, as expected. Figure $2 \mathrm{~b}$ shows the three-dimensional rendering of the raw $z$-stack images, as well as the corresponding deconvolution, as 
indicated (Supplementary Movie 1). Again, the protrusions in the cell membrane show more details in the deconvolved image. This result is more clearly demonstrated by investigating one slice from the tomogram, as shown in Fig. 2c, in which the empty space between spicules is revealed in greater detail after deconvolution.

\section{Tomography of Escherichia coli}

We further applied our approach to image E. coli cells. We acquired a $z$-stack consisting of 17 slices with a step size of $280 \mathrm{~nm}$. Figure $3 \mathrm{a}, \mathrm{b}$ shows the middle frame of raw data and the reconstructed $z$-stack. Upon deconvolution, the previously invisible protein helical subcellular structure of $E$. coli is resolved. These interesting structures have recently been investigated as a result of the development of high-resolution fluorescence microscopy techniques, with which the subcellular localizations of different proteins, such as the MinCD complex, FtsZ and MreB, are recorded. It has also been discovered that this helical structure is also related to lipopolysaccharides deposition ${ }^{34,46-48}$. The three-dimensional rendering of this E. coli cell is shown in Fig. 3c. Only the bottom half of the cell is shown in the figure to emphasize the helical subcellular structure (Supplementary Movie 2). Figure $3 \mathrm{~d}-\mathrm{f}$ presents profiles along the lines indicated in Fig. 3a-c.

\section{Tomography of HT29 cells}

To study more complex subcellular structures, we imaged human colon adenocarcinoma cells (HT29), using a $\times 63 / 1.4$ NA oil immersion objective. The $z$-stack, consisting of 140 frames, was acquired in $150 \mathrm{~nm} z$-steps. More details on the deconvolution algorithm are presented in the Supplementary Section $d$. The results obtained on a cell that has recently divided are summarized in Fig. 4. At the bottom of Fig. 4a,b we show specific regions of interest in $x-z$ and $x-y$ cross-sections. An increase in resolution is apparent in both the longitudinal (left, red box) and transverse (right, yellow box) directions. We used the deconvolved $z$-stack to generate the three-dimensional rendering of the HT-29 cell. WDT, like other quantitative phase imaging techniques, does not provide specificity, so subcellular structures are selected using a combination of features, including shape and refractive index. For example, nucleoli have the highest refractive indices in the cell and the nuclear membrane surrounding them typically reveals the cell nuclei. Figure $4 \mathrm{c}$ (Supplementary Movie 3) presents a false-colour three-dimensional image of an HT-29 cell, in which we can clearly observe the subcellular structures (cell membrane in blue; nuclei in green; nucleoli in red). These areas are first chosen by thresholding based on the phase values ( $0.6 \mathrm{rad}$ for nucleoli; $0.1 \mathrm{rad}$ for membrane) and then detailed based on morphology.

\section{Summary and discussion}

Our study shows that using spatially coherent and temporally incoherent light, three-dimensional structure information can be retrieved unambiguously, simply by scanning the focus through the object of microscope. This type of reconstruction requires a new theoretical description of the interaction between a weakly scattering object and broadband light, which we present here for the first time. In essence, our theory generalizes Wolf's diffraction tomography $^{7}$ to white light and correctly predicts that the sectioning capability is the result of the combined effect of coherence and high-NA gating. As a result, we can calculate the imaging system's response (PSF) and quantify the resolution, which turns out to be $350 \mathrm{~nm}$ transversally and $890 \mathrm{~nm}$ axially. Solving scattering problems using a quantitative phase microscope-that is, measuring at the image plane instead of the far-zone-is a powerful new concept that allows us to acquire light scattering data from completely transparent objects with high sensitivity and dynamic range. In practical terms, WDT renders three-dimensional images of unlabelled cells in the traditional environment of an inverted microscope, allowing for cell imaging over an extended period of time. Indeed, in the Supplementary Section g, we show HeLa cell three-dimensional imaging with WDT over a period of $24 \mathrm{~h}$. Accordingly, we anticipate that WDT will become a standard imaging modality in cell biology, complementing established technologies such as confocal microscopy.

\section{Methods}

Imaging. The specimen of interest was imaged with an inverted phase contrast microscope (Zeiss Axio Observer Z1) and white-light illumination. At the camera port, the microscope is equipped with a SLIM module, which renders quantitative phase images with subnanometre path-length sensitivity both spatially and temporally (see ref. 16 for details). To measure the axial dimension, the focal plane is scanned through the object by axially translating the objective lens (Fig. 5a). At each $z$-position of the object, a charge-coupled device (CCD) records the interferogram between the scattered $\left(U_{\mathrm{s}}\right)$ and unscattered $\left(U_{\mathrm{r}}\right)$ fields. For a more detailed description of the SLIM module, see Supplementary Fig. 1. The SLIM module introduces controllable phase shifts to $U_{r}$ in increments of $\pi / 2$ such that a unique quantitative phase image is reconstructed from four intensity images, as shown in Fig. $5 \mathrm{~b}$ and detailed in the Supplementary Section a. This acquisition process is repeated with an acquisition speed of 8 frames per second (f.p.s.), which translates to two quantitative images per second as the focus is scanned through the object by moving the objective lens, and the entire SLIM image $z$-stack is saved to disk for further processing. This acquisition speed is limited only by the detector frame rate and the refresh rate of the spatial light modulator. In the Supplementary Information we describe in detail the computer synchronization of data acquisition, as well as axial sampling and accuracy. Furthermore, we have previously demonstrated that SLIM can be used in parallel with other microscope imaging modalities such as fluorescence imaging, differential interference contrast, or bright-field ${ }^{16,22}$.

Therefore, combining these modalities with WDT can also be done in order to obtain three-dimensional high-resolution quantitative phase images with specificity. Because of its low exposure and phototoxicity, SLIM is capable of measuring over an extended period of time. Note that the environmental control is a standard accessory for the existing commercial microscope base. Accordingly, WDT can be used for four-dimensional imaging, with the fourth dimension being time. We provide more details on this capability in the Supplementary Section g.

It is clear from the raw $z$-stack data (Fig. $5 \mathrm{c}$ ) that optical sectioning is present in our quantitative phase images. However, to translate this phase information, which is a property of the optical field, into tomographic information, describing the object itself, we must develop a new inverse scattering theory to describe the white lightobject interaction. In essence, we extended the diffraction tomography calculations to white-light illumination and expressed the result in terms of the field crosscorrelation function, which is the measurable quantity. In the following, we provide the main steps in our derivation (a detailed derivation is provided in the Supplementary Section b).

Theory. As discussed in the Supplementary Section a, the measurable quantity in our phase shifting experiments is the temporal cross-correlation function $\Gamma_{12}$ between the scattered field $U_{\mathrm{s}}$ and the reference plane wave field $U_{\mathrm{r}}$ evaluated at the origin, that is, around the zero delay or $\tau=0 . \Gamma_{12}$ is defined as

$$
\Gamma_{12}(\mathbf{r}, \tau)=\left\langle U_{\mathrm{s}}(\mathbf{r}, t) U_{\mathrm{r}}^{*}(\mathbf{r}, t+\tau)\right\rangle
$$

where the asterisk denotes complex conjugation and the angle brackets denote ensemble averaging. The generalized Wiener-Khintchine theorem ${ }^{49,50}$ allows us to relate $\Gamma_{12}$ to the cross-spectral density, $W_{12}(\mathbf{r}, \omega)=\left\langle U_{\mathrm{s}}(\mathbf{r}, \omega) U_{\mathrm{r}}{ }^{\star}(\mathbf{r}, \omega)\right\rangle$, via a simple Fourier transform. Therefore, the measured cross-correlation at zero time delay can be expressed as an integral of the cross spectral density over all angular frequencies:

$$
\begin{aligned}
\Gamma_{12}(\mathbf{r}, \tau=0) & =\int_{0}^{\infty} W_{12}(\mathbf{r}, \omega) e^{-i \omega \tau} \mathrm{d} \omega \\
& =\int_{0}^{\infty}\left\langle U_{\mathrm{s}}(\mathbf{r}, \omega) U_{\mathrm{r}}^{*}(\mathbf{r}, \omega)\right\rangle \mathrm{d} \omega
\end{aligned}
$$

To calculate the integral in equation (5), we first derive a solution for the scattered field, $U_{s}$. The general scattering problem can be formulated by considering Fig. 1a. The Helmholtz equation, which describes the total field $U$, is given as

$$
\nabla^{2} U(\mathbf{r}, \omega)+\beta^{2}(\omega) U(\mathbf{r}, \omega)=-\chi(\mathbf{r}) \beta_{\mathrm{o}}^{2}(\omega) U(\mathbf{r}, \omega)
$$

where $\beta(\omega)=\bar{n} \beta_{0}(\omega)$, with $\bar{n}$ being the spatial average of the refractive index associated with the object $\left(\bar{n}=\langle n(\mathbf{r})\rangle_{\mathrm{r}}\right)$, which is assumed to be non-dispersive, $\beta_{0}(\omega)=\omega / c$ is the propagation constant (or the wavenumber) in vacuum, $\omega$ is the 
angular frequency, $\chi$ is the scattering potential of the non-dispersive object $\left(\chi(\mathbf{r})=n^{2}(\mathbf{r})-\bar{n}^{2}\right)$, and $n$ is the inhomogeneous refractive index associated with the object. $U$ can be written as the summation of the incident and scattered field, $U(\mathbf{r}, \omega)=U_{\mathrm{i}}(\mathbf{r}, \omega)+U_{\mathrm{s}}(\mathbf{r}, \omega)$, where $U(\mathbf{r}, \omega)=A(\omega) e^{i \beta(\omega) z}$ is the incident wave $(A(\omega)$ is the spectral amplitude of the incident field) and $U_{s}$ is the scattered wave, which is described by the reduced wave equation,

$$
\nabla^{2} U_{s}(\mathbf{r}, \omega)+\beta^{2}(\omega) U_{s}(\mathbf{r}, \omega)=-\chi(\mathbf{r}) \beta_{\mathrm{o}}^{2}(\omega) U(\mathbf{r}, \omega)
$$

The right-hand side of equation (7) is due to the scattering from the object, which acts as a secondary light source. We use the first-order Born approximation (see, for example, section 13.1.2. in ref. 4), which considers the scattering to be so weak, $\left|U_{\mathrm{s}}(\mathbf{r}, \omega)\right|<<\left|U_{\mathrm{i}}(\mathbf{r}, \omega)\right|$, that the field inside the object remains essentially a plane wave. This is a reasonable approximation in the context of imaging cells. Under these circumstances, on the right-hand side of equation (7) we can replace $U$ with $U_{\mathrm{i}}(\mathbf{r}, \omega)=A(\omega) e^{i \beta(\omega) z}$. Instead of using the traditional Green's function approach and Weyl's formula ${ }^{7}$, we solve this equation in the wavevector space (see chapter 2 in ref. 9). Thus, Fourier-transforming equation (7) with respect to $\mathbf{r}$ (see Supplementary Section b), we obtain the solution for the scattered field in the k-domain,

$$
U_{s}(\mathbf{k}, \omega)=-\beta_{0}^{2}(\omega) A(\omega) \frac{\chi\left[\mathbf{k}_{\perp}, k_{z}-\beta(\omega)\right]}{q^{2}\left(\mathbf{k}_{\perp}, \omega\right)-k_{z}^{2}}
$$

In equation (8), $\mathbf{k}=\left(k_{x}, k_{y}, k_{z}\right)$ is the wavevector, $\mathbf{k}_{\perp}=\left(k_{x}, k_{y}\right)$ is the transverse wavevector, and $q\left(\mathbf{k}_{\perp}, \omega\right)=\sqrt{\beta^{2}(\omega)-k_{\perp}^{2}}$. The denominator in equation (8) can be decomposed into two terms, $1 /\left[k_{z}-q(\omega)\right]$ and $1 /\left[k_{z}+q(\omega)\right]$, which correspond to the forward-scattering and back-scattering terms, respectively. Because our experiments are performed in transmission and we only measure forward-scattering waves, we retain the former term. We now take the inverse Fourier transform of equation (8) with respect to $k_{z}$. We therefore obtain the forward scattering field as a function of transverse wavevector $\mathbf{k}_{\perp}$, axial distance $z$ and angular frequency $\omega$ :

$$
U_{\mathrm{s}}\left(\mathbf{k}_{\perp}, z ; \omega\right)=-\frac{\beta_{0}^{2}(\omega) A(\omega) e^{i q z}}{2 q} \chi\left[\mathbf{k}_{\perp}, q-\beta(\omega)\right]
$$

Using the solution for $U_{s}$ from equation (9), we perform the integral in equation (5), which, after straightforward manipulations and change of variables (see Supplementary Section b), gives the following simple expression for the measured signal versus the transverse wavevector and axial distance,

$$
\Gamma_{12}\left(\mathbf{k}_{\perp}, z ; 0\right)=\Sigma\left(\mathbf{k}_{\perp}, z\right) \oplus_{z} \chi\left(\mathbf{k}_{\perp},-z\right)
$$

where $\oplus_{z}$ represents a convolution operation along the axial dimension $z$, between the object scattering potential $\chi$ and the instrument function $\Sigma$. More explicitly, the instrument function can be expressed as

$$
\Sigma\left(\mathbf{k}_{\perp}, z\right)=\frac{1}{8 \bar{n}^{2}} F T_{\mathrm{Q}}^{-1}\left[\frac{\left(Q^{2}+k_{\perp}^{2}\right)^{2}}{Q^{3}} S\left(-\frac{Q^{2}+k_{\perp}^{2}}{2 Q}\right)\right]_{z}
$$

Importantly, our theory uses no approximations on propagation (for example, no Fraunhoffer, or even Fresnel, approximations are used), which makes it suitable for high-resolution imaging.

\section{Received 26 February 2013; accepted 26 November 2013; published online 19 January 2014}

\section{References}

1. Als-Nielsen, J. \& McMorrow, D. Elements of Modern X-ray Physics (Wiley, 2001)

2. Jiang, H. et al. Quantitative 3D imaging of whole, unstained cells by using X-ray diffraction microscopy. Proc. Natl Acad. Sci. USA 107, 11234-11239 (2010).

3. Raines, K. S. et al. Three-dimensional structure determination from a single view. Nature 463, 214-217 (2010).

4. Born, M. \& Wolf, E. Principles of Optics: Electromagnetic Theory of Propagation, Interference and Diffraction of Light 7th expanded edn (Cambridge Univ. Press, 1999).

5. Devaney, A. J. Inverse-scattering theory within the Rytov approximation. Opt. Lett. 6, 374-376 (1981).

6. Wolf, E. in Advances in Imaging and Electron Physics Vol. 165 (ed. Hawkes, P. W.) Ch. 7 (Academic, 2011).

7. Wolf, E. Three-dimensional structure determination of semi-transparent objects from holographic data. Opt. Commun. 1, 153-156 (1969).

8. Gabor, D. A new microscopic principle. Nature 161, 777-778 (1948).

9. Popescu, G. Quantitative Phase Imaging of Cells and Tissues (McGraw-Hill, 2011).
10. Paganin, D. \& Nugent, K. A. Noninterferometric phase imaging with partially coherent light. Phys. Rev. Lett. 80, 2586-2589 (1998).

11. Marquet, P. et al. Digital holographic microscopy: a noninvasive contrast imaging technique allowing quantitative visualization of living cells with subwavelength axial accuracy. Opt. Lett. 30, 468-470 (2005).

12. Chalut, K. J., Brown, W. J. \& Wax, A. Quantitative phase microscopy with asynchronous digital holography. Opt. Express 15, 3047-3052 (2007).

13. Popescu, G. et al. Fourier phase microscopy for investigation of biological structures and dynamics. Opt. Lett. 29, 2503-2505 (2004).

14. Ikeda, T., Popescu, G., Dasari, R. R. \& Feld, M. S. Hilbert phase microscopy for investigating fast dynamics in transparent systems. Opt. Lett. 30, 1165-1168 (2005).

15. Popescu, G., Ikeda, T., Dasari, R. R. \& Feld, M. S. Diffraction phase microscopy for quantifying cell structure and dynamics. Opt. Lett. 31, 775-777 (2006).

16. Wang, Z. et al. Spatial light interference microscopy (SLIM). Opt. Express 19, 1016-1026 (2011).

17. Rappaz, B. et al. Comparative study of human erythrocytes by digital holographic microscopy, confocal microscopy, and impedance volume analyzer. Cytometry A 73A, 895-903 (2008).

18. Khmaladze, A., Kim, M. \& Lo, C. M. Phase imaging of cells by simultaneous dual-wavelength reflection digital holography. Opt. Express 16, 10900-10911 (2008).

19. Shaked, N. T., Rinehart, M. T. \& Wax, A. Dual-interference-channel quantitative-phase microscopy of live cell dynamics. Opt. Lett. 34, 767-769 (2009).

20. Park, Y. K. et al. Refractive index maps and membrane dynamics of human red blood cells parasitized by plasmodium falciparum. Proc. Natl Acad. Sci. USA 105, 13730-13735 (2008).

21. Park, Y. K. et al. Measurement of red blood cell mechanics during morphological changes. Proc. Natl Acad. Sci. USA 107, 6731-6736 (2010).

22. Mir, M. et al. Optical measurement of cycle-dependent cell growth. Proc. Natl Acad. Sci. USA 108, 13124-13129 (2011).

23. Pavillon, N. et al. Early cell death detection with digital holographic microscopy. PLoS ONE 7, e30912 (2012).

24. Chen, B. Q. \& Stamnes, J. J. Validity of diffraction tomography based on the first Born and the first Rytov approximations. Appl. Opt. 37, 2996-3006 (1998).

25. Carney, P. S., Wolf, E. \& Agarwal, G. S. Diffraction tomography using power extinction measurements. J. Opt. Soc. Am. A 16, 2643-2648 (1999).

26. Lauer, V. New approach to optical diffraction tomography yielding a vector equation of diffraction tomography and a novel tomographic microscope. J. Microsc. 205, 165-176 (2002).

27. Charriere, F. et al. Living specimen tomography by digital holographic microscopy: morphometry of testate amoeba. Opt. Express 14, 7005-7013 (2006).

28. Charriere, F. et al. Cell refractive index tomography by digital holographic microscopy. Opt. Lett. 31, 178-180 (2006).

29. Choi, W. et al. Tomographic phase microscopy. Nature Methods 4, 717-719 (2007).

30. Cotte, Y. et al. Marker-free phase nanoscopy. Nature Photon. 7, 113-117 (2013).

31. Choi, W. S., Fang-Yen, C., Badizadegan, K., Dasari, R. R. \& Feld, M. S. Extended depth of focus in tomographic phase microscopy using a propagation algorithm. Opt. Lett. 33, 171-173 (2008).

32. Zhou, R., Edwards, C., Arbabi, A., Popescu, G. \& Goddard, L. L. Detecting $20 \mathrm{~nm}$ wide defects in large area nanopatterns using optical interferometric microscopy. Nano Lett. 13, 3716-3721 (2013).

33. Goodman, J. W. Speckle Phenomena in Optics: Theory and Applications (Roberts \& Co., 2007).

34. Mir, M. et al. Visualizing Escherichia coli sub-cellular structure using sparse deconvolution spatial light interference tomography. PLOS ONE 7, e39816 (2012).

35. Wang, Z. et al. Spatial light interference tomography (SLIT). Opt. Express 19, 19907-19918 (2011).

36. Bon, P., Aknoun, S., Savatier, J., Wattellier, B. \& Monneret, S. in ThreeDimensional and Multidimensional Microscopy: Image Acquisition and Processing XX (eds Cogswell, C. J., Brown, T. G., Conchello, J.-A. \& Wilson, T.) 858-918 SPIE (2013).

37. Pawley, J. B. Handbook of Biological Confocal Microscopy 3rd edn (Springer, 2006).

38. Ding, H. F., Wang, Z., Nguyen, F., Boppart, S. A. \& Popescu, G. Fourier transform light scattering of inhomogeneous and dynamic structures. Phys. Rev. Lett. 101, 238102 (2008).

39. Chew, W. C. Waves and Fields in Inhomogeneous Media (IEEE Press, 1995).

40. Kim, T., Sridharan, S. \& Popescu, G. in Handbook of Coherent-Domain Optical Methods Vol. 1 (ed. Tuchin, V. V.) Ch. 7, 259-290 (Springer, 2013).

41. Huang, D. et al. Optical coherence tomography. Science 254, 1178-1181 (1991)

42. Ralston, T. S., Marks, D. L., Carney, P. S. \& Boppart, S. A. Interferometric synthetic aperture microscopy. Nature Phys. 3, 129-134 (2007).

43. Bain, B. J. A Beginner's Guide to Blood Cells 2nd edn (Blackwell, 2004). 
44. Khairy, K., Foo, J. \& Howard, J. Shapes of red blood cells: comparison of 3D confocal images with the bilayer-couple model. Cell. Mol. Bioeng. 1, 173-181 (2008).

45. Babacan, S. D., Wang, Z., Do, M. \& Popescu, G. Cell imaging beyond the diffraction limit using sparse deconvolution spatial light interference microscopy. Biomed. Opt. Exp. 2, 1815-1827 (2011).

46. Donachie, W. D. Co-ordinate regulation of the Escherichia coli cell cycle or the cloud of unknowing. Mol. Microbiol. 40, 779-785 (2001).

47. Raskin, D. M. \& De Boer, P. A. J. Rapid pole-to-pole oscillation of a protein required for directing division to the middle of Escherichia coli. Proc. Natl Acad. Sci. USA 96, 4971-4976 (1999).

48. Ghosh, A. S. \& Young, K. D. Helical disposition of proteins and lipopolysaccharide in the outer membrane of Escherichia coli. J. Bacteriol. 187, 1913-1922 (2005).

49. Wiener, N. Generalized harmonic analysis. Acta Mathematica 55, 117-258 (1930).

50. Khintchine, A. Eine verschärfung des poincaréschen 'wiederkehrsatzes'. Comp. Math 1, 177-179 (1935).

\section{Acknowledgements}

This research was supported in part by the National Science Foundation (grants CBET1040462 MRI, CBET 08-46660 CAREER) and the Science and Technology Center for

Emergent Behaviors of Integrated Cellular Systems (EBICS, CBET-0939511). The authors thank R. Bashir and K. Park for providing HT29 cells, I. Golding and M. Bednarz for providing E. coli cells and S. Robinson for assistance with SEM imaging of RBCs. The authors also thank J. Howard, K. Khairy and J.-J. Foo for providing confocal images of RBCs. R.Z. acknowledges support from the Beckman Foundation through a Beckman Graduate Fellowship. For more information, visit http://light.ece.illinois.edu

\section{Author contributions}

G.P., R.Z. and S.D.B. proposed the idea. G.P., R.Z., T.K. and P.S.C. developed the theoretical description of the method. T.K. and R.Z. performed three-dimensional PSF calculations. T.K. and M.M. performed quantitative phase imaging. S.D.B. and M.M. developed the sparse deconvolution method. T.K. and R.Z. performed data analysis and threedimensional reconstruction. G.P. and L.L.G. supervised the research. All authors contributed to writing the manuscript.

\section{Additional information}

Supplementary information is available in the online version of the paper. Reprints and permissions information is available online at www.nature.com/reprints. Correspondence and requests for materials should be addressed to G.P.

\section{Competing financial interests}

G.P. has financial interest in Phi Optics, Inc., a company developing quantitative phase imaging technology for materials and life science applications, which, however, did not sponsor the research.

\section{CORRIGENDUM}

\section{Demon-like algorithmic quantum cooling and its realization with quantum optics}

Jin-Shi Xu, Man-Hong Yung, Xiao-Ye Xu, Sergio Boixo, Zheng-Wei Zhou, Chuan-Feng Li, Alán Aspuru-Guzik \& Guang-Can Guo

Nature Photon. 8, 113-118 (2014); published online 19 January 2014; corrected after print 5 February 2014

In the version of this Letter originally published online and in print, the affiliation of Sergio Boixo was incorrectly given. He is affiliated with the Department of Chemistry and Chemical Biology, Harvard University, Cambridge, Massachusetts 02138, USA. This has now been corrected in the HTML and PDF versions of this Letter.

\section{CORRIGENDUM}

\section{Simultaneous measurement of nanoscale electric and magnetic optical fields}

B. le Feber, N. Rotenberg, D. M. Beggs and L. Kuipers

Nature Photonics 8, 43-46 (2014); published online: 15 December 2013; corrected after print: 30 January 2014

The reference section in the print version of this Letter contained the following errors:

For ref. 3, the volume number should have been 4 rather than 3 .

For ref. 17, "15, 1289-1295" should have been "http://dx.doi.org/10.1126/science.1232009".

For ref. 30, the volume number should have been 326 rather than 23 .

The online HTML and PDF versions of the Letter do not contain these errors. 